\title{
Supported Return to Training (The SuppoRTT Programme) -
}

\section{After almost a decade since Bawa-Garba: Has the culture changed?}

\author{
Sarah Siddiqui MBBS BDS BSc 1, Indranil Chakravorty PhD FRCP 2 \\ 1. SuppoRTT National Fellow, Health Education England London \\ 2. SuppoRTT Champion, St Georges University Hospitals NHS Trust, London
}

Correspondence to; sarah.siddiqui@hee.nhs.uk

\begin{abstract}
The Supported Return to Training programme (SuppoRTT) was established and funded in England by the Department of Health after recommendations from various professional bodies, trade union organisations and regulatory agencies. There may be a link between the awareness raised amongst the entire profession and the public by the tragic case of the loss of life of a young boy, and the SuppoRTT programme. Although organisations have signed up to the SuppoRTT programme, and resources have been made available, there are still challenges. Principal amongst them is the lack of information amongst trainees and trainers alike, as well as the culture of 'resilience or coping silently' with all eventualities that is inbred in the profession to its own detriment. The lack of a supportive culture amongst peers and a stressed workplace also contributes to burnout. This article explores the challenges and offers to increase awareness. Solutions and a change of hearts and minds will have to come from within the profession.
\end{abstract}

\section{Keywords}

Supported return to training; resilience; phased return; career break;

Cite as: Siddiqui S, Chakravorty I. (2020) Supported Return to Training (The SuppoRTT Programme) Almost a decade since Bawa-Garba: Has anything changed? The Physician 6(1):

epub 25.02.2020 (v1 Pre-print); final v2 04.05.2020 DOI: 10.38192/1.6.1.6

Peer Review: Reviewed

\section{Background}

Training to be a doctor begins formally as a young adult entering university, continues for half-a decade and then as a postgraduate learner, remaining the primary focus for several more years. The learning never quite stops, as the race to keep abreast of a rapidly evolving field of medical science, social expectations and culture keeps medical professionals on their feet and engaged in a lifelong quest. Combined with this are other factors: personal motivation to seek the best for every patient, empathy for the fellow human being, coping with a challenging environment of diminishing resources, rising demand for better health, an ageing population with multiple comorbidities, a health service frequently at the 
mercy of domestic politics, international conflict or economic downturns; thus creating a recipe for lifelong stress and burnout. (1)

Sometimes, a basic fact of life often escapes the attention of healthcare strategists and workforce planners, that parallel to the need for progress and competency for healthcare professionals; exists the need for emotional, social and financial stability in order to establish oneself in a traditional conservative profession balancing sometimes the start of a life relationship, or sometimes the demands of a young family.(2) Based on socio-cultural and educational norms in many societies, such 'burden/ responsibility' often falls disproportionately on women, who therefore find it seemingly more difficult to keep up with the demands of their chosen vocation or the oft punishing lifestyles of their peers. Often inadvertently ignored is personal health and well-being, therefore, contributing adversely to their own physical or mental health status.

When one takes a break from career or training, there is an artificial hiatus from the pace of learning and the adrenaline that keeps professionals constantly focused on progress and development. The common reasons for such career breaks can be parental leave, personal health related issues, family demands or more rarely as a consequence of medical errors or litigation. (2) Healthcare professionals who take parental leave identify a lack of an universal leave policy, strain of maintaining relentless training goals, loss of education time, lack of flexibility of programs and workplaces, and lack of support from faculty/peers as the major obstacles to taking leave during specialty training. (3)

More recently, the millennial generation have chosen to manage their own careers at their own pace or at a place of their choosing. This phenomenon became apparent after the regimentation of training programs was established and the rigidity of training pathways offered limited manoeuvring for individual tailoring or capacity to create temporal space for life events. (4) More than half of foundation doctors are taking a break after completion instead of directly entering specialty training programmes, choosing instead to do an "FY3", to explore a variety of specialties, go travelling or experience healthcare in distant lands.(5) Doctors within core and higher training are continuing the tradition of taking out of programme breaks for research, education or leadership roles. These phenomena throw up an increasing cohort of doctors who are now returning to practice after time away for various reasons.

\section{The Curious Case of Bawa-Garba}

For what is now probably the most influential case in the history of medical training in the UK, the tragic circumstances leading to the death of a young boy presenting with sepsis in 2011 and the sequelae for the involved healthcare professionals, shook the profession to its core. (6) Most attention has focused around the working environment, culture, support infrastructure, attrition of clinical skills during a period of break, senior supervision, gaps in the rota, unfamiliarity of the clinical environment among other factors which may have contributed to the tragedy. Dr Bawa Garba was sentenced for manslaughter, her licence erased from the register of medical practitioners and eventually partially restored. The focus of this article is not to debate the pros and cons of the case, nor how the regulator may have handled the investigation and the conflicts around the erasure, but to focus on the circumstances that may have left a young doctor vulnerable on her return to work after a period of parental leave. We know now that the support provided to this individual was non-existent. Hers was not an isolated incident, the story resonated with the entire frontline healthcare workforce.

Hundreds of doctors, nurses and other healthcare professionals are returning to clinical practice with little or no acknowledgement that they may have lost the sharpness of their clinical or procedural skills, that 
they may need a period of time to orient themselves with the much changed clinical environment and teams to regain their confidence. It is not anyone's fault, as most clinicians would not hesitate to agree that when away from frontline clinical work for prolonged periods, one loses one's speed and accuracy of decision-making. This attrition of skills is most easily quantifiable in surgeons and those that depend on procedural skills or physical dexterity. (7-9)

\section{Attrition of Skills}

The science of this recognised decay of clinical acumen, decision-making, accuracy of prescribing or dexterity in procedures is not well-documented barring few exceptions. Studies involving resuscitation where adherence to strict protocols may affect outcome or in specific surgical procedures (e.g. total hip replacement) show that periods of absence may adversely affect performance accuracy and recall. In nonsurgical disciplines, this attrition of skills is more challenging to measure and quantify. Although the evidence base is weak, most trainees and trainers agree that there is attrition and that a period of refresher/ retraining is necessary. (10)

\section{Rationale for SuppoRTT}

Although there is consensus in the profession that accuracy and comprehensiveness of professional education and training status (i.e. competency) does deteriorate during a period of absence, there is little evidence to suggest a minimum threshold for this loss. The rough consensus is around a period between 3-4 weeks (for certain highly skilled procedure dependent specialties) to 3 months before there is a noticeable change in performance. The attrition is also dependent heavily on the state of proficiency of the previously acquired skill before cessation (8) of practice and on the duration of the absence. It is expected that a doctor who has barely achieved minimum proficiency in a procedure or clinical decisionmaking will lose this skill quicker than one who has been practicing at an expert level for a longer period of time. (7) Hence on return, the more inexperienced learner will need more time and support than a more previously experienced professional. The bottom line is that the time and resources required for returning to expected proficiency is determined at an individual level, recognising the specialty and the clinical environment.

\section{Uptake and Awareness}

The SuppoRTT has been running for the last 2 years and funded by United Kingdom Department of Health (DoH) to the tune of GBf10 million.(11) Health Education England (HEE) has funded the appointment of champions within all NHS Trusts across England, set aside additional funding for a safe and supported return by means of refresher courses, funding for a period of enhanced supervision, coaching and mentorship. Each HEE Specialty School has appointed leads for developing the framework for delivery of minimum standards for safe return to clinical practice and education supervisor upskilling to better supervise the needs of this group is being delivered across England. (11)Yet, in the first 2 years of available data suggests that the uptake of the additional resources and funding in different regions, ranges from none, to a quarter of returning trainees. There are several factors that may be contributing to this; namely, a lack of awareness both amongst trainees and their supervisors, poor information or database infrastructure within human resource (HR) departments, inability for $H R$ databases to exchange information about the absence of trainees adequately with the training programmes and vice versa.

In the health sector there exists a perception that one would be judged unfairly, that one may appear weak and incompetent if asking for additional help. This is particularly true for those returning from nonparental leave and in traditionally demanding specialties e.g. surgery, cardiology etc. For international 
healthcare staff, they may be subject to prejudices, have their own cultural deference to authority, come from systems with less support which may pose additional challenges, i.e reluctance in asking supervisors for the action planning meetings, for additional resources, and the implications that may have on their career progression in an environment that may already contain a bias. There is a culture of being stoic and "just getting on with it", but in the event of an inadvertent clinical error, one is often faced with a punitive face of that culture which has often ended up holding a trainee negligent for systemic failings.(12)

\section{Challenges}

There are several hurdles that will need to be understood and addressed if this system were to be successful in achieving a safe and supported return to practice for all healthcare professionals. Educational programs also face challenges: cost of development and maintenance; allocation of staff and faculty time to re-educate returning physicians alongside other learners; provision of emotional support, counselling and career guidance; interpretation of varied licensing guidelines; and the need to tailor one's program to individual trainees. In some specialties there may be a number of sub-specialties with varying needs, and others the various grades of the returning trainees can pose a problem for pitching the course at the right level. Years out of practice and increasing physician age are predictors of poor performance.

The major themes that often emerge from the analysis of interviews from healthcare professionals returning are: compromise and feeling valued. The experience of returning to work is a process of compromise in which staff find strategies to cope with their changing roles vs demands, to find a balance between home vs work life. Staff want to feel valued by their managers and co-workers, as this enables them to feel comfortable, confident with some of the compromises they made.(13) Another challenge is in relation to physical and mental health related absences, where the assessment and intervention of an occupational health $(\mathrm{OH})$ team is critical to success. The $\mathrm{OH}$ teams may have to prescribe phased return and limitations on activities as well as duration. Integrating such specific requirements to a demanding schedule may be particularly challenging as it would be to provide additional on-site supervision. (14)

The elephant in the room is the large proportion of doctors who are not in training posts (often overseas qualified doctors), moving between training programmes or specialty schools or even between UK NHS regions (SuppoRTT is only available in England). There is no system or framework to recognise this large 'fluid population' of doctors and provide any oversight, supervision or support. At various workshops the discussion has often revolved around 'who should be responsible?'. The answer is often that it has to be the doctor who requires this support. No one would deny that and often the awareness of the deterioration in skills, competence and confidence is most acute in the doctor who is faced with this prospect. But these are the doctors who are most likely to be vulnerable, in fear of being considered incompetent or unsafe, or of losing their hard earned reputation as a reliable professional. The stakes are usually high and the odds often steeped against them. (15-17)

While there are now emerging frameworks for doctors who are returning after breaks, there are little structured resources to support nurses, midwives, paramedics and other frontline healthcare staff. The General Medical Council and Nursing \& Midwifery council are starting to set up requirements for registrants returning after career breaks. Other registration bodies such as the General Pharmaceutical Council or the Healthcare Professions should follow soon. 
There may be registration requirements, funding sources for trainee doctors and for all staff in statutory parental leave with Keep in Touch (KiT) days, but who will provide the funding needed for non-trainee doctors, consultants, nurses, midwives and allied health professionals who may be in rotational appointments or shorter contracts. None of the NHS Trust finances are in a healthy position to support the additional funding needed for supernumerary or phased return. Yet we must consider that at the receiving end of any delay or inaction in this area will be the unsuspecting patient who may suffer consequences of a healthcare professional whose clinical acumen and skill has decayed through a planned or unplanned break. However, one must not forget the consequences on the 'second victim' - the professional themselves.(12)

There also remains the challenge of the low uptake and awareness. If there is an underspend in the funding due to poor awareness and implementation by trusts, supervisors and trainees, as with most budgets in healthcare, there is a risk of it being taken away for causes deemed more noteworthy.

\section{Conclusion}

The only proper way to view this challenge of facilitating doctors (and all healthcare professionals) to take career pauses or breaks and to return them safely back to practice would be from the lens of the patient receiving treatment by the ethical principles of 'do no harm' as most critical decisions in healthcare are based on. Once the culture of safety, blame free learning and planned risk avoidance becomes mainstay, the funding and resources will follow. SuppoRTT champions have a key role to play in the near future to develop frameworks, set minimum standards and monitor progress towards a safe and supportive NHS for all.

\section{CASE STUDIES}

"Deena is an oncology registrar, who has taken time off to be a carer for her elderly father who has had a long battle with cancer and has tragically passed away. She has done an odd shift in A\&E as a locum during this time. She is due to return to training in 2 months in the same hospital where her father was treated. She cannot begin to contemplate how this will feel to walk into those rooms without him and see the same healthcare professionals who treated him and how she will adjust to becoming the clinician treating patients from being on the other side. She is uncertain how to raise this with her educational supervisor in case it impacts on her career progression, she already feels behind due to her time out.

"Jyoti is an ST4 in ENT, who had to take time out of training to have emergency cardiothoracic surgery. She has recently tried to get in touch with her last clinical supervisor and training programme director to indicate her clinician is happy for her to return to training. She has had 11 months of sickness absence and is concerned about her level of deskilling and how she will cope with returning to a completely new workplace and how supportive her supervisors will be"

\section{Questions to consider}

- In your educational supervisor role, how would you assess these trainee's needs and prepare an action plan for their return? Who else would need to be involved?

- As a clinical supervisor how could you facilitate informing your department managers and rota coordinators in time so they can access the supernumerary funding and make suitable adjustments to accommodate the trainees safe return to training? 
- As the training programme director how would you in your role support the trainee's transition in terms of navigating the effect on training time, highlighting the SuppoRTT process and funding and other resources the trainee could make use of?

- As a trainee stepping out of training, who would you contact to get more information and support and how can you enable yourself to have the right conversations with the key individuals who can make a positive difference to your return to training?

\section{References}

1. Lemaire JB, Wallace JE. Burnout among doctors. BMJ [Internet]. 2017 Jul 14 [cited 2020 Jan 21];358. Available from: https://www.bmj.com/content/358/bmj.j3360

2. Kärkkäinen R, Saaranen T, Hiltunen S, Ryynänen OP, Räsänen K. Systematic review: Factors associated with return to work in burnout. Occup Med Oxf Engl. 2017 Aug 1;67(6):461-8.

3. Altieri MS, Salles A, Bevilacqua LA, Brunt LM, Mellinger JD, Gooch JC, et al. Perceptions of Surgery Residents About Parental Leave During Training. JAMA Surg. 2019 Aug 7;

4. Patel F, Chakravorty I. Millennials - The missing piece in the NHS Workforce puzzle? Sushruta [Internet]. 2020 Jan 21 [cited 2020 Feb 24];13(1). Available from: https://www.sushruta.net/vol-13-1-Mar2020/Workforce

5. Scanlan GM, Cleland J, Johnston P, Walker K, Krucien N, Skåtun D. What factors are critical to attracting NHS foundation doctors into specialty or core training? A discrete choice experiment. BMJ Open. 2018 Mar 1;8(3):e019911.

6. The Bawa-Garba case | The BMJ [Internet]. [cited 2020 Jan 21]. Available from: https://www.bmj.com/bawa-garba

7. Jones GF, Forsyth K, Jenewein CG, Ray RD, DiMarco S, Pugh CM. Research Residents' perceptions of skill decay: Effects of repeated skills assessments and scenario difficulty. Am J Surg. 2017 Apr;213(4):6316.

8. Gawad N, Allen M, Fowler A. Decay of Competence with Extended Research Absences During Residency Training: A Scoping Review. Cureus [Internet]. [cited 2020 Feb 24];11(10). Available from: https://www.ncbi.nlm.nih.gov/pmc/articles/PMC6874279/

9. D’Angelo A-LD, Ray RD, Jenewein CG, Jones GF, Pugh CM. Residents' perception of skill decay during dedicated research time. J Surg Res. 2015 Nov;199(1):23-31.

10. Lammers RL, Davenport M, Korley F, Griswold-Theodorson S, Fitch MT, Narang AT, et al. Teaching and assessing procedural skills using simulation: metrics and methodology. Acad Emerg Med Off J Soc Acad Emerg Med. 2008 Nov;15(11):1079-87.

11. SuppoRTT [Internet]. Health Education England. 2017 [cited 2020 Jan 31]. Available from: https://www.hee.nhs.uk/our-work/supporting-doctors-returning-training-after-time-out

12. Radhakrishna S. Culture of blame in the National Health Service; consequences and solutions. BJA Br J Anaesth. 2015 Nov 1;115(5):653-5.

13. Brightwell A, Minson S, Ward A, Fertleman C. Returning to clinical training after maternity leave. BMJ [Internet]. 2013 Oct 9 [cited 2020 Jan 31];347. Available from: https://www.bmj.com/content/347/bmj.f5965

14. Kärkkäinen R, Saaranen T, Räsänen K. Occupational health care return-to-work practices for workers with job burnout. Scand J Occup Ther. 2019 Apr;26(3):194-204.

15. BMA - Staffing crisis in NHS laid bare, as new BMA analysis shows that three quarters of medical specialities face shortage of doctors [Internet]. [cited 2020 Jan 21]. Available from: https://www.bma.org.uk/news/media-centre/press-releases/2017/september/staffing-crisis-in-nhslaid-bare 
16. Hashim A. Educational challenges faced by international medical graduates in the UK. Adv Med Educ Pract. 2017;8:441-5.

17. Woolf K, Rich A, Viney R, Needleman's, Griffin A. Perceived causes of differential attainment in UK postgraduate medical training: a national qualitative study. BMJ Open. 2016 25;6(11):e013429. 